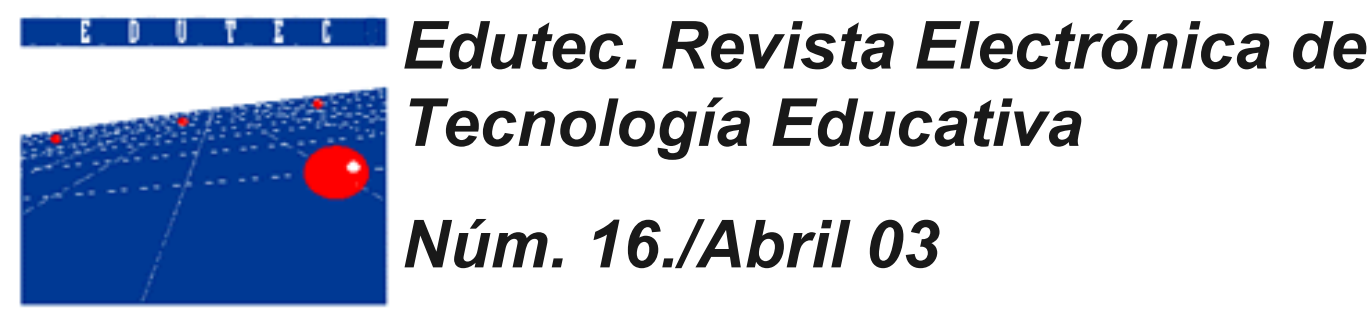

\title{
LOS MÉTODOS PRODUCTIVOS Y SU VINCULACIÓN CON EL ORDENADOR. "UNA EXPERIENCIA EN CIRCUITOS ELECTRÓNICOS I"
}

Lic. José León Montoya

Universidad de Oriente

Facultad de Ingeniería Eléctrica

Departamento de Telecomunicaciones y Electrónica

ileon@fie.uo.edu.cu

\section{Introducción :}

Los imperativos y demandas para incorporar las nuevas tecnologías de la información y comunicación, y particularmente el ordenador, en el sistema educativo, en el mundo de las escuelas y el que hacer pedagógico de los profesores, cobran día a día más relieve.

Nuestro entorno social, cultural, laboral y profesional está siendo seriamente reconstruido como consecuencia de la denominada revolución informática, y, ciertamente, la escuela no puede dar la espalda a esa realidad social y a las demandas de diverso signo que plantea. Hoy por hoy, sin embargo, la presencia de las nuevas tecnologías en nuestras aulas y enseñanza es muy escasa, probablemente insignificante. La cacareada "Revolución Informática" no ha llegado de forma importante y generalizada a nuestro sistema escolar, encargados socialmente de organizar, codificar, tratar y representar la información y el conocimiento para los alumnos.

La consideración de la utilización del ordenador en la Educación es un tema de encuentro para muchos investigadores, diseñadores, responsables y administradores de la Educación, y también, por supuesto, algunos profesores. En mucho de los contextos en que se abordan - aunque naturalmente depende de quienes sean los sujetos implicados y cuales sus intereses tienden a generar una suerte de futurismo cargados de promesas y grandes realizaciones. Como han resumido Miller y Olson (1992), el ordenador y la Educación a puesto en circulación nuevos lemas y el augurio de grandes posibilidades educativas. Se aventura, así, una revolución en profundidad de la Educación, la más importante en el siglo XVI. Con respecto a los alumnos y su aprendizaje se habla de las enormes posibilidades asociadas a la creación de micromundos, entornos de aprendizaje, o sistemas interactivos multimedias, que pueden estimular y cultivar no sólo habilidades de pensamiento difícilmente alcanzables con otros medios, sino también un contexto de aprendizaje más rico, estimulante; creativo y potenciador de su autonomía para aprender a aprender, tratar y representar la información con posibilidades insospechadas, desarrollar habilidades y destrezas, comunicarse con otros, etc. (Bartolomé Pina 1992; de Castro Lozano y otros 1992; Sancho, 1992)

Cuando se piensa en el profesor, algunos, lo más considerados con el mismo, prevén que la introducción del ordenador en su trabajo pedagógico, como un recurso para el desarrollo de la enseñanza, va a realizar serias transformaciones que enriquecerán y diversificaran sus funciones de sus "Resistencia al cambio" llegan poco más allá de asignarle un papel de mero supervisor del 
aprendizaje de los alumnos, liberado de las tareas más penosas y rutinarias vinculadas a la medición y control de lo que van adquiriendo. Incluso algunos, los más optimista y convencidos del poder de ciertos programas de ordenador para cultivar capacidades formales de pensamiento en los alumnos, escépticos con respecto al saber y la capacidad de los profesores, auguran que serán relegados al desempeño de tareas meramente ejecutivas y gerenciales. Una tarea importante a realizar en este sentido, será la de capacitarlos para que realicen justamente aquellas tareas que no dificulten las posibilidades inherentes a los "nuevos programas de ordenador" para el desarrollo de las capacidades más insospechadas de razonamiento abstracto, inferencia, tratamiento de datos, resolución de problemas, etc.

\section{Desarrollo:}

Podemos agrupar los distintos métodos en dos grandes grupos según Lener y Skatkin.

\begin{tabular}{|l|l|}
\hline $\begin{array}{l}\text { 1. Métodos reproductivos (El alumno se apropia de } \\
\text { conocimientos y reproduce los ya conocidos). }\end{array}$ & $\begin{array}{l}\text { - Método explicativo ilustrativo o informativo } \\
\text { receptivo } \\
\text { - Método reproductivo }\end{array}$ \\
\hline
\end{tabular}

\section{Exposición problémica}

2. Métodos productivos (El alumno alcanza nuevos conocimientos como resultado de la actividad creadora)

- Método de búsqueda parcial o heurístico. - Método investigativo

Podemos considerar un grupo intermedio, al cual pertenece la exposición problémica, pues supone la asimilación tanto de información elaborada, como de elementos de la actividad creadora.

Esta división de los métodos no está aislados uno de otros. Esta división de los métodos en productivos es relativa, ya que es imposible concebir la actividad creadora sin la actividad reproductora y viceversa; el acto de reproducción de conocimientos contiene elementos creadores en cuanto a la organización lógica de la exposición, entre otras cosas.

Al resolver cualquier problema, el hombre actualiza y mentalmente reproduce los conocimientos que ya posee y al aplicarlos de sus propias variantes a partir del proceso de elaboración creadora que ha realizado

La utilización de los métodos ha de caracterizarse por su flexibilidad. En dependencia con los objetivos, el contenido del tema el nivel de desarrollo y preparación de los alumnos variarán o se alterarán los métodos durante la clase.

\section{Método investigativo}

Para llegar a la asimilación de los conocimientos en el tercer nivel y asimilar íntegramente la experiencia de la actividad creadora, es necesario emplear el método investigativo.

Algunos pedagogos consideran este método universal de aprendizaje.

El método garantiza el dominio de las vías del conocimiento científico, forma los rasgos de la actividad creadora, despierta el interés por este tipo de actividad y además ofrece conocimientos integrales.

En esencia, el método investigativo es el método de organización de la actividad de búsqueda de los alumnos, tendente a solucionar problemas cuya solución aún desconocen.

La mayor parte de las actividades investigativas deben constituir pequeñas tareas de búsqueda que exigen, sin embargo, recorrer todas o la mayoría de las etapas del proceso de investigación.

Estas etapas son:

1. La observación y el estudio de los hechos y fenómenos. 
2. Esclarecimiento de los fenómenos sujetos a investigación, que no resulten claros y comprensibles.

3. Formulación de Hipótesis.

4. Elaboración del Plan de investigación.

5. Ejecución del Plan consistente en esclarecer los vínculos de lo que estudia con otros fenómenos.

6. Formulación de los resultados.

7. Comprobación de los resultados.

8. Conclusiones sobre la posible y necesaria aplicación de los conocimientos adquiridos.

Nos referimos a investigaciones sencillas de carácter docente.

La actividad del maestro consiste en organizar aquellas actividades que aseguren la aplicación creadora por parte de los alumnos de los conocimientos necesarios para la solución de la incógnita, desarrollando capacidades, hábitos y habilidades necesarios al trabajo científico.

La aplicación del principio del aumento gradual de las dificultades durante el proceso docente educativo, nos obliga a ir representando gradualmente a los alumnos las distintas etapas a seguir en el conocimiento científico, es por ello que el sistema de métodos analizados se ponen al servicio del cumplimiento de este objetivo.

El método de exposición problémica así como el de búsqueda parcial o heurística, preceden y acompañan al método investigativo propiamente dicho. Lo preceden, cuando los alumnos carecen aún de experiencia para resolver los problemas en su totalidad y lo acompañan, cuando es necesario apropiarse de la experiencia de un tipo nuevo y complejo de problemas, o cuando ayudan a aclarar y solucionar un problema cuya solución resultó difícil a los alumnos.

Las actividades o tareas inherentes al método investigativo son múltiples y su realización puede ser en clase o extraclase.

Ejemplo de los mismos son: La elección de materiales de consulta, apuntes, resúmenes, confección de fichas bibliográficas, elaboración del plan de investigación etc.

A continuación se explicará las formas en que se pretende utilizar el ordenador en el análisis de circuitos eléctricos en Ingeniería, así como se ilustrarán varios ejemplos en los cuales se explican de forma breve el cumplimiento de los métodos de enseñanza utilizando como soporte material de los mismos el ordenador.

\section{Vinculación con el Ordenador}

La introducción del Ordenador en Circuitos Eléctricos I responde a la consecución de tres metas.

1. Fomentar la cultura informática.

2. Promover la creatividad individual y el sentido de confianza a través del diseño y el producto de programas.

3. Mejorar la capacidad del pensamiento, con particular énfasis sobre los procesos de análisis y síntesis.

La idea Básica de la aplicación del ordenador en Circuitos Eléctricos I da respuesta a tres preguntas.

¿Dónde?

Se debe hacer un estudio de los temas que forman el sistema de conocimientos de la asignatura, para poder determinar de forma precisa en aquellas cuestiones que por el nivel de complejidad y el 
volumen excesivo de operaciones matemáticas, se adaptan para la utilización del ordenador. Tratando de reducir las operaciones auxiliares y empleando ese tiempo para incrementar la actividad creadora del alumno.

¿Cuándo?

- Sé este familiarizado con los programas de simulación que utilizará para desarrollar las distintas actividades.

- Sea capaz de seleccionar los problemas que se deben de resolver con el ordenador y cuales no se deben resolver en el ordenador, en este sentido el estará guiado en todo momento por el profesor.

- A través de una solución o varias soluciones de un problema relativamente sencillo estos guiados por el profesor lleguen a definir, comprobar leyes, teoremas y conceptos que para ello van a constituir un conocimiento nuevo.

- Se necesite la experimentación y tomas de decisiones sobre situaciones que en realidad pueden ser peligrosas.

¿Cómo?

\section{Primera Etapa:}

Actividades en el ordenador que le permiten al estudiante familiarizarse con los programas de simulación con los que ellos van a trabajar en clases.

\section{Segunda Etapa:}

Actividades en las cuales el estudiante planteará el sistema de ecuaciones necesarias para dar solución al circuito a el asignado. Con este sistema el cual se obtiene a partir de la aplicación de un método de solución de circuitos resistivos puros y utilizando un programa de simulación matemática resolverá dichas ecuaciones obteniendo así el resultado buscado.

Aquí estamos logrando que él sea capaz de seleccionar y aplicar el método adecuado para la solución del circuito, como también ejercite el planteamiento de las ecuaciones circuitales necesarias según el método seleccionado. Ver Anexo 1

\section{Tercera Etapa:}

Actividades en las cuales el estudiante simulará en el ordenador el circuito designado para esto el tiene que ser capaz de preparar su circuito, así como todos los datos que debe de suministrar al programa, para esta actividad debe utilizar un programa de simulación de Circuitos Eléctricos obteniendo los resultados como parte de la simulación los cuales tendrá que interpretar físicamente y comparar con los resultados de la etapa anterior. También con dichos resultados se pueden comprobar el cumplimiento de leyes, principios y teoremas circuitales haciendo siempre un análisis físico de dicha comprobación.

Es posible obtener nuevas corridas de dicho programa haciendo cambios de parámetros en los datos suministrado, incluso para situaciones límites peligrosas, interpretando físicamente los nuevos resultados obtenidos. Ver anexo 2.

\section{Cuarta Etapa:}

Actividades en las cuales se hace referencia a la actuación del ordenador como mediador entre el Alumnos y un cierto modelo de un Circuito Eléctrico real o de una situación concreta. El programa introduce al alumno en un proceso progresivo de aprendizaje mediante revelaciones que la simulación proporciona. En este tipo de actuación se otorga una mayor importancia a lo conceptual que lo informativo. Por ello se utiliza el término de revelatorio, ya que el significado de los conceptos es revelado al alumno mediante el empleo de técnicas y situaciones que facilitan la exploración, el descubrimiento y el desarrollo de la intuición. Aquí el aprendizaje se realiza al efectuar descubrimientos y establecer relaciones. Ver Anexo 3.

\section{Conclusiones}


1. Como vemos los objetivos fundamentales del trabajo de investigación se cumplen ya que se brinda una recopilación de métodos y programas que conforman la base científica y programoteca del departamento de Electrotecnia Básica en el área de la computación aplicada a la solución de circuitos eléctricos y electrónicos.

2. En el trabajo de investigación se logra recomendar la implementación de un sistema metodológico de aplicación de las técnicas de computación, en la disciplina de circuitos eléctricos. Se concluye que la aplicación de la computación será el método empleado, esto se planteo como medio para que el estudiante aprecie que la computación es un medio importantísimo pero no sustituye el fenómeno estudiado en sí.

3. Esperamos que este trabajo brinde un material de estudio que pueda servir en alguna medida, para abordar todo lo relacionado con la aplicación de la computación en circuitos eléctricos, como forma de mejorar el proceso de enseñanza aprendizaje en las CES en los cuales se imparte la asignatura.

\section{Bibliografía}

1. Skatkin y Danilov. "Didáctica de la Escuela Media". Editorial de libros para la Educación. La Habana 1978.

2. S. Tolbtov V.N. "El Maestro, la Ciencia Moderna y los Métodos de enseñanza". Editorial de libros para la Educación. La Habana 1977.

3. Bossuet. G: "La Computadora en la Escuela". Editorial Gedisa. Barcelona 1985.

4. Delval, J: Crecer y Pensar la construcción del conocimiento en la Escuela. Eitorial Laira. Barcelona 1983.

5. Delval, J: Niños y Máquinas: Los ordenadores en la Educación. Editorial. Alianza. Madrid 1986.

6. Fernández González; M: Enseñanza asistida por Ordenador. Editorial Anaya. Madrid 1983.

7. Garcia Ramos, L y Ruis, F: Informática y Educación. Panorama, aplicaciones y perspectivas. Editorial García Ramos. Barcelona 1984.

\section{Anexo 1}

Ejemplo que ilustra las actividades en el ordenador según se explica en la Segunda Etapa:

- El alumno recibirá un esquema de circuitos eléctricos y de acuerdo con el método de las corrientes de mallas y el método de los potenciales de nodo después; planteará todas las ecuaciones que permitan determinar el estado eléctrico del circuito.

Esta tarea constituye la tarea de entrada sin lo cual no podrá obtener el derecho a realizar el trabajo en el centro de cálculo.

- Con los sistemas de ecuaciones necesarios, se trasladará al centro de cálculo (Laboratorio de Ordenadores) y empleando un programa de simulación matemática resolverá el sistema de ecuaciones planteado por el método de las corrientes de mallas, primeramente y posteriormente resolverá el sistema no planteado para el método de los potenciales de nodos. También podrá utilizar las máquina como calculadora en el caso que lo requiera.

- Con los valores obtenidos, comprobarán las leyes de Kirchhoff (L.K.C y L.K.V) luego determinará mediante el procedimiento de cálculo necesario el balance de potencias del circuito queremos señalar que el alumno debe llevar al centro de cálculo todas las ecuaciones que le permitan determinar las corrientes por las ramas; comprobar las leyes de Kirchhoff y hacer el balance energético, dejando los espacios necesarios para colocar los valores que ira obteniendo. 
- Teniendo en cuenta los resultados obtenidos durante la clase se confeccionará un informe.

- Luego discutirá los resultados con el profesor.

\section{Anexo 2}

Ejemplo que ilustra las actividades en el ordenador según se explica en la Tercera Etapa.

- Usted recibirá un esquema de circuito eléctrico y seguidamente procederá a resolver el circuito seleccionado utilizando un programa de simulación de circuitos eléctricos, teniendo en cuenta todos los requerimientos que exige este programa, en cuanto a la configuración topológica del circuito y el análisis de corriente directa que se llevará a cabo, así como las magnitudes que se quieren obtener con la simulación realizada.

- Primeramente se le pedirá al programa todas las corrientes de ramas y después también se obtendrán los potenciales de cada modo con respecto a una referencia dada.

- Obtenidos los potenciales de cada nodo el estudiante calculará las corrientes de ramas comparando estos resultados con los que el programa determino anteriormente.

- Teniendo en cuenta que el análisis se realizará para la variación de todas las fuentes de voltaje en $\pm 50 \%$ de su valor y la variación del valor de $R 6=0$ y $R 6= \pm 50 \%$ de su valor lo cual quiere decir que se realizarán 7 análisis. En cada análisis el alumno comprobará el cumplimiento de las leyes de Kirchhoff (L.K.C y L.K.V) como también se realizará el balance energético en cada caso.

- Confeccionará las conclusiones sobre los resultados obtenidos para las variaciones de loa elementos.

\section{Anexo 3}

Ejemplo que ilustra las actividades en el ordenador según se explica en la Cuarta Etapa.

- Usted recibirá un esquema de circuito eléctrico y seguidamente procederá a graficar el circuito seleccionado utilizando programas de simulación de circuitos eléctricos.

- Primeramente se le pedirá al programa los potenciales de cada nodo con respecto a una referencia dada, luego se procederá a cambiar varias veces el nodo de referencia y dada esta situación determinar nuevamente el potencial de cada nodo.

\section{Cuestionario}

1) ¿El potencial de cada nodo depende de las referencia escogida en el circuito?

¿Por qué?

- Después se le pedirá al programa la diferencia de potencial entre dos nodos considerando que en el circuito existe un nodo de referencia, luego se procederá a cambiar varias veces el nodo de referencia y dada esta situación determinar nuevamente la diferencia de potencial.

\section{$\underline{\text { Cuestionario }}$}

2) ¿La diferencia de potencial de cada rama del circuito eléctrico estudiado depende de la referencia escogida?

¿Por qué?

- Luego se le pedirá al programa la corriente que circula en cada rama del circuito, luego se escogerá una rama determinada y se procederá a disminuir la resistencia de la rama, y luego aumentaremos esta misma resistencia. 


\section{Cuestionario}

3) ¿La corriente de la rama seleccionada depende del valor de la resistencia? ¿Por qué?

- Si se aumenta el valor de la fuente de alimentación y se piden las corrientes de ramas del circuito.

\section{Cuestionario}

4) ¿Las corrientes de ramas en el circuito dependen del aumento de la fuente de alimentación?

¿Por qué?

Trabajo de Investigación

Se orientará por parte del profesor tareas de investigación en las cuales los estudiantes realizaran de forma independiente con ayuda del ordenador para obtener los resultados deseados.

\section{Ejemplos}

Hipótesis

¿Es posible en un circuito eléctrico de corriente directa lograr que exista máxima transferencia de potencia entre una fuente real y una carga?

\section{En el circuito eléctrico representado}

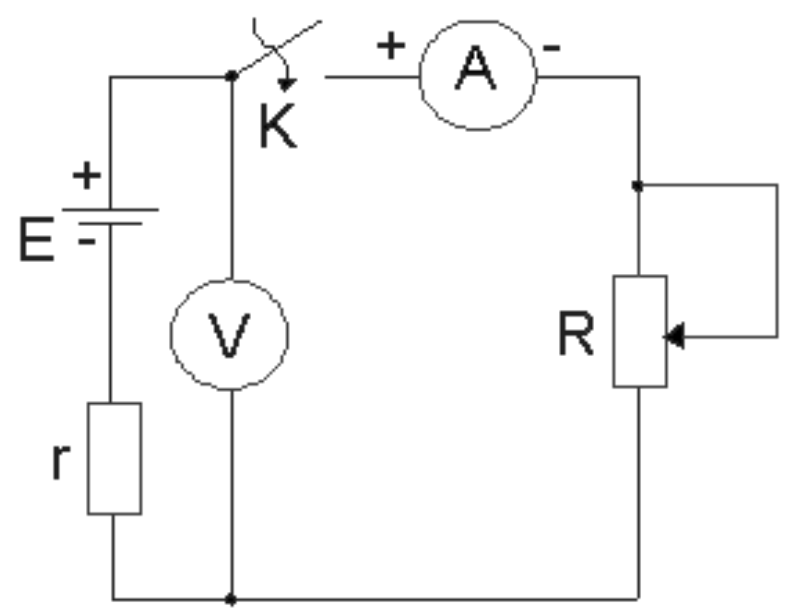

- Utilizando un programa de simulación adecuado

1. ¿Determine la lectura de los instrumentos cuando el interruptor $\mathrm{K}$ esta abierto?

2. ¿Determine la lectura de los instrumentos cuando el interruptor $\mathrm{K}$ esta cerrado?

- ¿En ambos casos los resultados obtenidos son iguales?

¿Por qué?

3. ¿Considerando que la $\mathrm{R}$ de carga es cero y $\mathrm{K}$ cerrado determine los valores de los instrumentos?

¿El valor de la corriente del circuito que valores tendrán?

¿Por qué? 
¿Calcule con los valores obtenidos la potencia útil del circuito?

4. ¿Considerando que $R$ de la carga es igual a $r$ interna de la fuente y $\mathrm{K}$ cerrado determine los valores de los instrumentos?

¿El valor de la corriente del circuito que valores tendrían?

¿Por qué?

¿Calcule con los valores obtenidos la potencia útil del circuito?

5. ¿Considerando que $\mathrm{R}$ de la carga es mayor a la $\mathrm{r}$ interna de la fuente y $\mathrm{K}$ cerrado determine los valores de los instrumentos?

¿El valor de la corriente del circuito que valores tendrían?

¿Por qué?

¿Calcule con los valores obtenidos la potencia útil del circuito?

¿Calcule con los valores obtenidos en todos casos anteriores la eficiencia del circuito?

- Plotear un sistema de coordenadas donde se ilustre una representación gráfica de las lecturas de los instrumentos para los casos estudiado (V contra I).

- Plotear un sistema de coordenada donde se ilustre una representación gráfica de los valores calculados de potencia útil de la fuente contra la lectura del amperímetro para los casos estudiados (P útil contra I)

- Plotear un sistema de coordenadas donde se ilustre una representación gráfica de los valores calculados de eficiencia de la fuente contra la lectura del amperímetro para los casos estudiados ( $\mathrm{h}$ contra I)

Estos gráficos se deben construir con un programa de simulación adecuado, teniendo todos estos resultados.

- Confirmar valides de la hipótesis.

- ¿Qué condición debe cumplirse en el circuito para que ocurra lo confirmado?.

- ¿Qué implicaría dicha condición en cuanto a la eficiencia del circuito?

- ¿Es posible obtener una misma potencia con dos corrientes diferentes?

- ¿En caso de escoger entre esta corrientes cual usted tomaría?

¿Por qué?

- Confirmar valides de la hipótesis.

- ¿Qué condición debe cumplirse en el circuito para que ocurra lo confirmado?.

- ¿Qué implicaría dicha condición en cuanto a la eficiencia del circuito?

- ¿Es posible obtener una misma potencia con dos corrientes diferentes?

- ¿En caso de escoger entre esta corrientes cual usted tomaría?

¿Por qué? 